\title{
VORTEX-INDUCED VIBRATION OF CATENARY RISER: REDUCED-ORDER MODELING AND LOCK-IN ANALYSIS USING WAKE OSCILLATOR
}

\author{
Narakorn Srinil * \\ Centre for Applied Dynamics Research \\ Department of Engineering, King's College \\ University of Aberdeen \& MCS \\ Scotland, UK \\ Patrick O’Brien \\ MCS \\ Aberdeen, Scotland, UK
}

\begin{abstract}
A novel reduced-order fluid-structure interaction model for the vortex-induced vibration of catenary riser subject to the ocean current is developed and systematically investigated. The semi analytical-numerical approach accommodates multi-mode nonlinear dynamic responses and accounts for the effect of varying initial curvature of the inclined flexible cylinder. The geometrically nonlinear equations of riser motion are based on a pinned-pinned beam-cable model with bending and extensibility stiffness. The empirical hydrodynamic model is based on a distributed van der Pol wake oscillator which approximates the space-time varying fluid forces. In this initial study, the incoming current flow is assumed to be steady, uniform, unidirectional and perpendicular to the riser initial plane of curvatures. Thus, emphasis is placed on evaluating the riser cross-flow responses due to fluctuating lift forces. A preliminary validation of model and analysis results has been performed. Several insights into the vortex-induced vibration of catenary risers are highlighted through a series of parametric studies. These include the characterization of single-mode vs. multimode lock-in, the limitations of a single-mode solution through a convergence analysis which accounts for a varying number of considered riser modes, the prediction of riser maximum response amplitudes, the quantitative/qualitative behaviors of tension- or beam-dominant catenary risers and the overall influence of fluid-riser parameters. Moreover, recent industrial concepts of modes switching/sharing are discussed along with the meaningful effect of Reynolds number.
\end{abstract}

\author{
Marian Wiercigroch \\ Centre for Applied Dynamics Research \\ Department of Engineering, King's College \\ University of Aberdeen \\ Scotland, UK \\ Rae Younger \\ MCS \\ Aberdeen, Scotland, UK
}

\section{INTRODUCTION}

Ongoing deepwater applications in oil \& gas industry have demonstrated the need to thoroughly understand the nonlinear dynamic behaviors of a steel catenary riser (SCR) undergoing vortex-induced vibration (VIV) due to the ocean current. Apart from the significant effects of surface waves, floating vessel motions and seabed interactions, VIV is thought to be the single most crucial factor that should be taken into account as a potential cause of fatigue damage for SCRs. Nevertheless, many insightful VIV aspects of SCRs remain unanswered. Indeed, through lack of a general, reliable and computationally-efficient fluid-riser interaction model which accounts for both inherent fluid mechanics and nonlinear physics of the curved flexible cylinders, several uncertainties take place throughout the SCR dynamic analysis, design and construction process, respectively.

The awareness of SCR technology has advanced through recent numerical and experimental investigations which unveil some meaningful VIV behaviors of SCRs (e.g., Vandiver and Gonzalez 1997; Hatton and Willis 1998; Lie et al. 2001, Moe et al. 2004; de Lima et al., 2007). In spite of this, the industrial VIV analysis tool is still based on an empirical science with some ad hoc approximations. Owing to the impractical timeconsuming 3-D flow visualizations by CFD for a long slender structure, the VIV fluid excitation and damping forces typically rely on the hydrodynamics data obtained from a laboratory testing of an elastically-supported rigid cylinder vibrating with 1 or 2 degrees of freedom (DOF) in a uniform flow at a low Reynolds (Re) number.

* Corresponding author: NarakornSrinil@mcs.com 


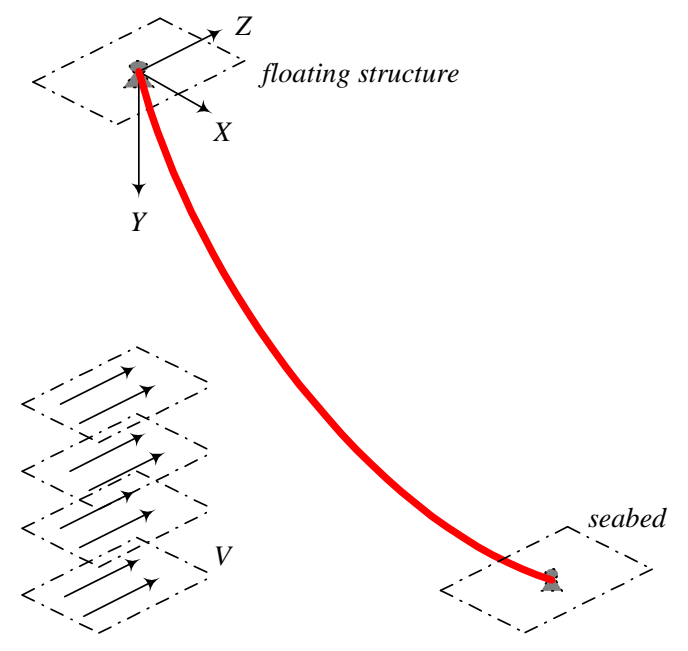

Figure 1 Schematic model of SCR subject to a uniform current

An investigation into the vortex shedding patterns and the fundamental 3-D wake topology for the flow past a stationary curved circular cylinder has been carried out by Miliou et al. (2003). As a result of pipe curvatures, the computational simulations highlight different kinds of wake characteristics depending on the pipe (convex or concave) configuration and its orientation with respect to (aligned with or normal to) the incoming flow. When the flow is uniform and normal to the curvature plane, the cross-flow wake dynamics of curved pipes behave qualitatively similar to those of straight pipes. This is in contrast to the case of flow being aligned with the curvature plane where wake dynamics change dramatically. For this reason, in this initial investigation, the current flow approaching the SCR is assumed to be steady, unidirectional, uniform and perpendicular to the curvature plane of the inclined cylinder (see Figure 1). This avoids the multiplicity of vortex shedding frequencies (e.g., the case of flow aligned with the curvature plane) which would complicate the modeling and analysis. Emphasis is placed on the cross-flow VIV due to fluctuating lift forces occurring solely in the SCR curvature plane with horizontal/vertical dynamic responses.

This study aims to develop a numerically-robust reducedorder fluid-structure interaction model capable of analyzing the nonlinear dynamic responses of SCRs subject to VIV and describing the associated single-mode vs. multi-mode lock-in. With respect to the typical riser model based on the linear equation of a straight tensioned-beam motion, a more realistic curved beam-cable (bending-extensibility) model - accounting for the meaningful effect of varying curvature in the static equilibrium of SCRs - is considered. As large dynamic displacements are likely to take place for slender underwater structures with high aspect ratios and potential multi-mode interactions, the effect of geometrical nonlinearities is also taken into account. The empirical hydrodynamic model is based on a nonlinear van der Pol wake oscillator model whose theoretical background is briefly summarized in the following.

\section{NONLINEAR WAKE OSCILLATOR MODEL}

The simplest and computationally robust means to reestablish the hydrodynamic forcing caused by the periodic vortex shedding behind a rigid or elastic circular cylinder is to employ a phenomenological wake oscillator (see a recent review by Parkinson 1989; Gabbai and Benaroya 2005). Typically, the wake oscillator is based on the nonlinear van der Pol equation having a term (terms) coupled with a structural equation of motion such as the linear spring-mass-damping oscillator. Relevant parameters and coefficients are obtained by calibrating through experimental data. Unfortunately, the wake oscillator does not capture at all the flow field physics and almost all of the models reported in literature to date are restricted to the lift force governing cross-flow VIV. The measurement set-up is usually rearranged such that the drag force governing in-line VIV is negligible or uncoupled with cross-flow VIV. Nevertheless, the wake oscillator proves to be useful for describing a self-limiting nature of VIV responses observed by many experiments and flow visualizations. In addition, the lock-in or synchronization regime can be captured and readily ascertained (e.g., Cunha et al. 2006).

Recently, the van der Pol oscillator for VIV has been revised by Skop and Balasubramanian SB (1997), Facchinetti, de Langre and Biolley FLB (2004) in order to overcome some limitations of previous wake oscillators. Both models capture the self-limiting amplitude responses at zero structural damping and reproduce some qualitative, as well as quantitative, aspects of VIV when compared with experiment results. Regarding the application to flexible cylinders, the SB model has been used in the analysis of single-mode cross-flow/in-line VIV of suspended cables (Kim and Perkins 2002) and SCRs (Srinil et al. 2008). The FLB model has been considered by Violette et al. (2007) for the cross-flow VIV of long tensioned-beam and straight cable (string model). They showed a good comparison with direct numerical simulations and experiments.

In this study, the SB model is adapted for the multi-mode cross-flow VIV of SCRs. The space-time varying lift coefficient $C_{L}(s, t)$ and corresponding wake oscillator $Q(s, t)$ are originally expressed as

$$
\begin{aligned}
& C_{L}(s, t)=Q(s, t)-\frac{2 \gamma}{\omega_{S}} \dot{Y}_{N}(s, t), \\
& \ddot{Q}(s, t)-\omega_{S} G\left(C_{L 0}^{2}-4 Q^{2}(s, t)\right) \dot{Q}(s, t)+\omega_{S}^{2} Q(s, t)=\omega_{S} F \dot{Y}_{N}(s, t),
\end{aligned}
$$

in which $\gamma$ is a stall parameter, $C_{L 0}$ is the lift coefficient of a stationary cylinder, a dot denotes differentiation with respect to time $t$. Herein, $Y_{N}$ is defined as a local riser displacement normal to its tangential axis and $s$ denotes arc-length coordinate along riser. By considering the spatially uniform flow normal to SCR curvature plane with a speed $V$, the vortex-shedding frequency $(\mathrm{rad} / \mathrm{s})$ in the wake $\left(\omega_{S}\right)$ is unique such that $\omega_{S}=$ $2 \pi \mathrm{St} V / D$, where $D$ is the hydrodynamic diameter and $\mathrm{St}$ is the Strouhal number. The empirical coefficients $F$ and $G$ play a meaningful role in the overall wake-riser interaction responses. 


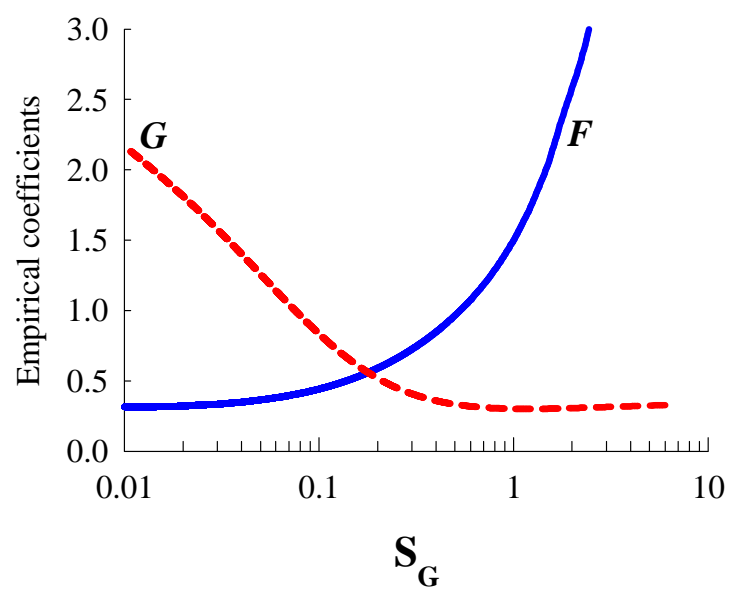

Figure 2 Variation of empirical coefficients with $S_{G}$ parameter

Following Srinil et al. (2008), to account for the effect of SCR initial curvatures and to describe the concurrent horizontal $(u)$ and vertical $(v)$ displacement components of SCR in-plane $(X-Y)$ motion (Figure 1), we project $Q_{X}=-Q \sin \theta\left(u=-Y_{N} \sin \theta\right)$ and $Q_{Y}=Q \cos \theta\left(v=-Y_{N} \cos \theta\right)$. The space-dependent local angle $\theta$ is measured clockwise from the horizontal $X$-axis and can be analytically obtained from the SCR static equilibrium analysis. Consequently, equation (2) entails

$$
\begin{aligned}
& \frac{\ddot{Q}_{X}}{\sin \theta}-\frac{\omega_{S} G C_{L 0}^{2} \dot{Q}_{X}}{\sin \theta}+\frac{4 \omega_{S} G Q_{X}^{2} \dot{Q}_{X}}{\sin ^{3} \theta}+\frac{\omega_{S}^{2} Q_{X}}{\sin \theta}=\frac{\omega_{S} F \dot{u}}{\sin \theta} \\
& \frac{\ddot{Q}_{Y}}{\cos \theta}-\frac{\omega_{S} G C_{L 0}^{2} \dot{Q}_{Y}}{\cos \theta}+\frac{4 \omega_{S} G Q_{Y}^{2} \dot{Q}_{Y}}{\cos ^{3} \theta}+\frac{\omega_{S}^{2} Q_{Y}}{\cos \theta}=\frac{\omega_{S} F \dot{v}}{\cos \theta}
\end{aligned}
$$

In contrast to equation (2), above nonlinear wake oscillators are now dependent on both time and space variables. $Q_{X}$ and $Q_{Y}$ are to be determined together with $u$ and $v$, whereas $C_{L 0}$ is a given constant. The wake coefficients $(F, G)$ are obtained by matching a series of experimental data which generally include the measurement of the maximum response amplitude of cylinder $A / D$ and the vortex-to-structural frequency ratio during VIV (see, Skop and Balasubramanian 1997). As exemplified in Figure 2, $F$ and $G$ are dependent on the system mass-damping (so-called Skop-Griffin) parameter $\mathrm{S}_{\mathrm{G}}=\xi / \mu$, in which $\xi$ is the modal damping of riser and $\mu$ is the mass ratio given by

$$
\mu=\frac{\rho D^{2}}{8 \pi^{2} \mathrm{St}^{2}\left(m+m_{a}\right)},
$$

where $\rho$ is the fluid density, $m$ is the riser mass/length and $m_{a}$ denotes the potential added mass/length $\left(m_{a}=C_{A} \rho A_{r}\right.$, with $A_{r}$ being the hydrodynamic cross-sectional area and $C_{A}=1$ ).

It is worth remarking that, albeit capturing some effects of fluid and structural material properties, the SB model does not account for the meaningful effect of Re number as recently highlighted by experimental observations (Swithenbank et al. 2008). In other words, $F$ and $G$ in Figure 2 are unchanged when varying $V(\mathrm{Re})$ in a lock-in analysis (Srinil et al. 2008). To incorporate such Re number effect in the derivation of $F$ and $G$, one may utilize a recent empirical formula given by Govardhan and Williamson (2006) which reads

$$
A / D=\left(1-1.12 \alpha+0.30 \alpha^{2}\right) \log \left(0.41 \mathrm{Re}^{0.36}\right)
$$

where the associated mass-damping parameter is $\alpha=\left(m^{*}+C_{A}\right) \xi$ and the mass ratio $m^{*}=m /\left(\pi \rho D^{2} / 4\right)$. As an example, with $\mathrm{S}_{\mathrm{G}}=$ 0.227 and corresponding $\alpha=0.091$, the SB model provides the fixed $F$ and $G$ equal to 0.644 and 0.489 (Srinil et al. 2008), respectively. By accounting for equation (6), $F$ and $G$ are varied depending on the Re number, as exemplified in Table 1.

\section{Table 1 Effect of Re number on wake coefficients}

\begin{tabular}{|r|r|r|}
\hline $\mathrm{Re}$ & $F$ & $G$ \\
\hline 5000 & 0.641 & 0.597 \\
\hline 10000 & 0.644 & 0.470 \\
\hline 25000 & 0.647 & 0.357 \\
\hline 50000 & 0.648 & 0.297 \\
\hline 75000 & 0.649 & 0.268 \\
\hline 100000 & 0.650 & 0.251 \\
\hline 250000 & 0.651 & 0.205 \\
\hline
\end{tabular}

It can be seen that the Re number substantially (slightly) affects the wake $G(F)$ coefficient. $G$ decreases with increasing Re, highlighting how the nonlinear damping effect - regulating the self-limiting response or limit cycle - decreases (see equations 3, 4). Accordingly, the maximum structural response amplitude is expected to increase with increasing Re. This insightful aspect - captured by the underlying wake oscillator model - provides qualitative agreement with experiment results and theoretical explanation (Govardhan and Williamson 2006; Swithenbank et al. 2008). The Re-effect on VIV responses of SCRs will be highlighted and discussed in Section 5.

\section{NONLINEAR EQUATIONS OF RISER MOTION}

With reference to the global Cartesian coordinate system, Figure 1 displays a continuum model of arbitrarily sagged and inclined SCR connected from a stationary floating structure to a seabed with pinned-pinned supports. The steady incoming flow is in the $Z^{+}$-direction perpendicular to the SCR plane $(X Y)$ of initial curvatures. A horizontal offset $X_{H}$ and water depth $Y_{H}$ define a chord inclination angle of SCR (i.e., $\theta_{r}=\tan ^{-1} Y_{H} / X_{H}$ ).

For convenience in the theoretical modeling which relies upon continuous functions, it is assumed that a 2-D submerged static configuration of SCR is solely due to its effective self weight and is described by a closed-form, hyperbolic functionbased, catenary formula (Srinil et al. 2008). The bending restraint and current flow action are considered to play a role after the completion of static equilibrium. Such neglected static bending is plausible as boundary conditions are pinned-pinned and the SCR curvatures are relatively small. 
By considering the SCR as a flexural elastic curved beamcable with the Euler-Bernoulli hypothesis, the geometrically nonlinear partial-differential equations of SCR in-plane motion about its equilibrium may be expressed in a general dimensional form as (Srinil et al. 2008)

$$
\begin{aligned}
& \left(m+m_{a}\right) \frac{\partial^{2} u}{\partial t^{2}}+c \frac{\partial u}{\partial t}=\frac{\partial}{\partial s}\left\{T\left(\frac{\partial u}{\partial s}\right)+E A_{r}\left(\frac{\partial x}{\partial s} \frac{\partial u}{\partial s}+\frac{\partial y}{\partial s} \frac{\partial v}{\partial s}+\right.\right. \\
& \left.\left.\frac{1}{2}\left(\left(\frac{\partial u}{\partial s}\right)^{2}+\left(\frac{\partial v}{\partial s}\right)^{2}\right)\right)\left(\frac{\partial x}{\partial s}+\frac{\partial u}{\partial s}\right)-E I \frac{\partial}{\partial s}\left(\frac{\partial^{2} u}{\partial s^{2}}\right)\right\}+H_{1}, \\
& \left(m+m_{a}\right) \frac{\partial^{2} v}{\partial t^{2}}+c \frac{\partial v}{\partial t}=\frac{\partial}{\partial s}\left\{T\left(\frac{\partial v}{\partial s}\right)+E A_{r}\left(\frac{\partial x}{\partial s} \frac{\partial u}{\partial s}+\frac{\partial y}{\partial s} \frac{\partial v}{\partial s}+\right.\right. \\
& \left.\left.\frac{1}{2}\left(\left(\frac{\partial u}{\partial s}\right)^{2}+\left(\frac{\partial v}{\partial s}\right)^{2}\right)\right)\left(\frac{\partial y}{\partial s}+\frac{\partial v}{\partial s}\right)-E I \frac{\partial}{\partial s}\left(\frac{\partial^{2} v}{\partial s^{2}}\right)\right\}+H_{2},
\end{aligned}
$$

in which $s$ denotes Lagrangian coordinate, $u(x)$ and $v(y)$ represent dynamic (static) displacement in the horizontal $(X)$ and vertical $(Y)$ direction, respectively. Riser properties are considered to be spatially uniform, with mass $(m)$, added mass $\left(m_{a}\right)$, structural damping coefficient $(c)$, bending $(E I)$ and axial $\left(E A_{r}\right)$ stiffness. $T$ denotes axial static tension of riser and $H_{i}$ denotes hydrodynamic forces leading to cross-flow VIV. By accounting for both bending and axial extensibility effects, equations $(7,8)$ are also valid for a straight top-tensioned riser (TTR) or a horizontal pipeline with zero sag $(x=y=0)$, and accounts for overall inertial effects through quadratic/cubic nonlinearities (Srinil and Rega 2007b). The effects of shear, torsion, seabed and internal flow-induced friction forces, which are quite important for SCRs, are not herein considered.

As the current flow is normal to the SCR plane, the crossflow VIV due to lift force $F_{L}$ corresponds to the SCR in-plane motion. Thus, by neglecting the tangential hydrodynamics, the excitation forces per unit length in equations $(7,8)$ read

$$
\begin{aligned}
& H_{1}=-F_{L} \sin \theta=-\frac{1}{2} \rho C_{L} D V^{2} \sin \theta, \\
& H_{2}=F_{L} \cos \theta=\frac{1}{2} \rho C_{L} D V^{2} \cos \theta,
\end{aligned}
$$

where $C_{L}$ is the lift coefficient based on equation (1) and wake oscillators, equations $(3,4)$. It is worth noting that the mean component of the lift coefficient, which is usually trivial for straight cylinders, takes place for curved cylinders (Miliou et al. 2003). This potentially gives rise to a new SCR equilibrium during VIV. Yet, this aspect is omitted as attention is particularly paid on the fluctuating component. For convenience in the parametric studies, the arc-length $s$ coordinate is projected onto the coordinate $x$ which is, in the following, considered as a new independent parameter. The differential equations $(3,4)$ and $(7,8)$ are rearranged in the temporal firstorder system. Overall displacement variables, together with associated equations, are normalized with respect to $D$.

\section{REDUCED-ORDER MODEL FOR MULTI-MODE VIV}

This paper is aimed at numerically and systematically investigating the multi-mode VIV responses of SCRs through a reduced-order fluid-riser interaction model having a few DOF. Owing to geometrical and hydrodynamic nonlinearities, it is assumed that the coupled riser-wake responses contain spatial contributions from a certain number of modes whose natural frequencies are commensurable to vortex-shedding frequency. These modes are defined as potentially vortex-excited modes. The multi-mode response aspect is realistic since a slender continuum structure has multiple natural frequencies whose values are closely spaced and occasionally in a nearly integer ratio (e.g., 1:1, 2:1 or even 2:2:1). Consequently, by assuming a standing wave characteristic of marine riser responses, both displacement $(u, v)$ and associated velocity (defined as $\left.A_{i}, B_{i}\right)$ variables in equations $(3,4)$ and $(7,8)$ are postulated through a series of $N$ in-plane SCR linear modes as follow

For riser dynamics,

$$
\begin{aligned}
& \dot{u}=A_{1} \rightarrow \quad u=\sum_{n=1}^{N} f_{n} \phi_{n}, \quad A_{1}=\sum_{n=1}^{N} p_{n} \phi_{n}, \\
& \dot{v}=A_{2} \rightarrow \quad v=\sum_{n=1}^{N} f_{n} \varphi_{n}, \quad A_{2}=\sum_{n=1}^{N} p_{n} \varphi_{n} .
\end{aligned}
$$

For wake dynamics,

$$
\begin{aligned}
& \dot{Q}_{x}=B_{1} \rightarrow \quad Q_{x}=\sum_{n=1}^{N} d_{n} \phi_{n}, \quad B_{1}=\sum_{n=1}^{N} e_{n} \phi_{n}, \\
& \dot{Q}_{y}=B_{2} \rightarrow \quad Q_{y}=\sum_{n=1}^{N} d_{n} \varphi_{n}, \quad B_{2}=\sum_{n=1}^{N} e_{n} \varphi_{n} .
\end{aligned}
$$

where $\phi_{n}$ and $\varphi_{n}$ are horizontal and vertical displacement components of $n^{\text {th }}$ mode shape functions associated with natural frequencies $\omega_{n}$ of the submerged riser. These are obtained based on a Fourier sine basis in conjunction with a hybrid analyticalnumerical solution of linear equations of the free undamped motion in $(7,8)$ with bending/extensibility (Srinil et al. 2008). In equations $(11,12), f_{n}\left(d_{n}\right), p_{n}\left(e_{n}\right)$ are generalized coordinates of riser (wake) to be determined. By substituting equations (11, 12) into $(3,4)$ and $(7,8)$, performing the standard Galerkin procedure with zero displacements and curvatures at end boundaries, and applying the orthonormalization of modes, a system of nonlinear coupled riser-wake equations describing the multi-mode lock-in $\left(\omega_{s} \approx \omega_{n}\right)$ is expressed as

$$
\begin{aligned}
\dot{f}_{n}= & p_{n}, \\
\dot{p}_{n}= & -2 \xi_{n} \omega_{n} p_{n}-\omega_{n}^{2} f_{n}+\mu \omega_{n}^{2}\left(d_{n}-\frac{2 \gamma}{\omega_{s}} p_{n}\right)+\sum_{i=1}^{N} \sum_{j=1}^{N} \Lambda_{n i j} f_{i} f_{j} \\
& +\sum_{i=1}^{N} \sum_{j=1}^{N} \sum_{k=1}^{N} \Gamma_{n j i k} f_{i} f_{j} f_{k}, \\
\dot{d}_{n}= & e_{n}, \\
\dot{e}_{n}= & \omega_{s} G C_{L 0}^{2} e_{n}-\omega_{s}^{2} d_{n}+\omega_{s} F p_{n}+ \\
& \sum_{\substack{i=1, i \neq n}}^{N} \Pi_{n i}\left(\omega_{s} G C_{L 0}^{2} e_{i}-\omega_{s}^{2} d_{i}+\omega_{s} F p_{i}\right)-4 \omega_{s} G \sum_{i=1}^{N} \sum_{j=1}^{N} \sum_{k=1}^{N} \Re_{n i j k} d_{i} d_{j} e_{k},
\end{aligned}
$$


in which quadratic/cubic nonlinear coefficients - accounting for multi-mode contributions and interactions - read

$$
\begin{gathered}
\Lambda_{n i j}=-c_{r} \int_{0}^{X_{H} / D} \frac{1}{k^{3}}\left(\frac{3}{2} \phi_{n}^{\prime} \phi_{i}^{\prime} \phi_{j}^{\prime}+y^{\prime} \phi_{n}^{\prime} \phi_{i}^{\prime} \varphi_{j}^{\prime}+\frac{1}{2} \phi_{n}^{\prime} \varphi_{i}^{\prime} \varphi_{j}^{\prime}+\varphi_{n}^{\prime} \phi_{i}^{\prime} \varphi_{j}^{\prime}+\right. \\
\left.\frac{y^{\prime}}{2} \varphi_{n}^{\prime} \phi_{i}^{\prime} \phi_{j}^{\prime}+\frac{3}{2} y^{\prime} \varphi_{n}^{\prime} \varphi_{i}^{\prime} \varphi_{j}^{\prime}\right) d x, \\
\Gamma_{n i j k}=-\frac{c_{r}}{2} \int_{0}^{X_{H} / D} \frac{1}{k^{3}}\left(\phi_{n}^{\prime} \phi_{i}^{\prime} \phi_{j}^{\prime} \phi_{k}^{\prime}+\phi_{n}^{\prime} \phi_{i}^{\prime} \varphi_{j}^{\prime} \varphi_{k}^{\prime}+\varphi_{n}^{\prime} \phi_{i}^{\prime} \phi_{j}^{\prime} \varphi_{k}^{\prime}+\varphi_{n}^{\prime} \varphi_{i}^{\prime} \varphi_{j}^{\prime} \varphi_{k}^{\prime}\right) d x, \\
\Pi_{n i}=\int_{0}^{X_{H} / D} k\left(\frac{\phi_{n} \phi_{i}}{y^{\prime}}+\varphi_{n} \varphi_{i}\right) d x / \int_{0}^{X_{H} / D} k\left(\frac{\phi_{n}^{2}}{y^{\prime}}+\varphi_{n}^{2}\right) d x, \\
\Re_{n i j k}=\int_{0}^{X_{H} / D} k^{3}\left(\frac{\phi_{n} \phi_{i} \phi_{j} \phi_{k}}{y^{3}}+\varphi_{n} \varphi_{i} \varphi_{j} \varphi_{k}\right) d x / \int_{0}^{X_{H} / D} k\left(\frac{\phi_{n}^{2}}{y^{\prime}}+\varphi_{n}^{2}\right) d x .
\end{gathered}
$$

Here, a dash denotes differentiation with respect to $x, k=$ $\left(1+y^{\prime 2}\right)^{1 / 2}, c_{r}=E A_{r} /\left(m+m_{a}\right) D^{2}$. Equations (17-20) are numerically integrated based on a 64-point Gaussian quadrature. Depending on the number of considered riser $N$ modes, the total $N^{2}\left(N^{3}\right)$ quadratic (cubic) coefficients in each $n$ equation are calculated a priori, as discussed by Srinil et al. (2009). Equation (16) entails $N$ coupled van der Pol oscillators with identical vortex frequency $\omega_{s}$. They are, in turn, coupled with the nonlinear structural oscillators through associated $p$-terms. The $4 N$ equations (13-16) are simultaneously solved by a numerical integration method with a stable time stepping and properly assigned initial conditions.

Overall, the VIV response of riser depends on mass ratiodamping parameter, static configuration profile, modal shape functions, system vortex/natural frequencies, strength of fluidstructure nonlinearities and empirical wake $(F, G)$ coefficients. Through lack of actual SCR experimental data associated with each considered $n^{\text {th }}$ mode, $F$ and $G$ are considered to be constant or mode-independent (Figure 2) for a given $V$. Yet, they may be varied with varying $V$ if one accounts for also the Re number effect through equation (6), as discussed in Table 1.

\section{PARAMETRIC RESULTS AND DISCUSSION}

By way of examples, both cable- and beam-dominant SCRs are analyzed. The riser material-geometric properties may be characterized by a single non-dimensional flexural-extensibility parameter which reads (Srinil et al. 2008)

$$
\Delta=L \sqrt{T_{a} / E I},
$$

in which $L$ is the SCR equilibrium length and $T_{a}$ is the SCR tension at maximum sag. For TTRs, $L$ is the span length and $T_{a}$ is the uniform (or averaged) tension. $\Delta$ describes how the flexural (small $\Delta$ ) or axial (large $\Delta$ ) rigidity plays a predominant role. Based on riser data provided by Srinil et al. (2008) and the joint industry research programme STRIDE (Moe et al. 2004), some associated parameters meaningful in the VIV analysis are given in Table 3 as SCR1 and SCR2, respectively.
Table 2 Parameters of considered SCRs

\begin{tabular}{|r|r|r|}
\hline Parameters & SCR1 & SCR2 \\
\hline$\Delta$ & 272 & 21 \\
\hline$L / D$ & 2581 & 835 \\
\hline$\theta_{r}$ & $30^{\circ}$ & $37.62^{\circ}$ \\
\hline$\mu$ & .044 & .121 \\
\hline$\xi_{n}$ & $1 \%$ & $1 \%$ \\
\hline $\mathrm{S}_{\mathrm{G}}$ & .227 & .082 \\
\hline$\alpha$ & .091 & .033 \\
\hline$c_{r}$ & $1.3 \times 10^{8}$ & $2 \times 10^{10}$ \\
\hline $\mathrm{sag} / \mathrm{span}$ & .082 & .024 \\
\hline$F$ & .644 & .432 \\
\hline$G$ & .489 & .982 \\
\hline
\end{tabular}

Note that parameters $C_{L 0}=0.28, \gamma=0.183, \mathrm{St}=0.2$ are fixed in both SCR cases. The damping ratios $\xi_{n}$ (thus $\mathrm{S}_{\mathrm{G}}, F, G$ ) of all considered modes are assumed to be equal since emphasis herein is placed on investigating the multi-mode coupling effect resulting from the (curved or straight) riser geometry. The cable-dominant SCR1 $\left[\Delta \approx O\left(10^{3}\right)\right]$ has larger aspect ratio $L / D$ (meaningful for deepwater application), sag/span and $S_{\mathrm{G}}$ than the beam-dominant SCR2 $\left[\Delta \approx O\left(10^{2}\right)\right]$.

To ascertain the occurrence of (single-mode or multi-mode) lock-in and predict the maximum response amplitudes when varying $V$, it is common in practice to make a reference to a reduced flow velocity parameter $U_{r}$ which is defined by

$U_{r}=\frac{2 \pi V}{\omega_{n r} D}$

where $\omega_{n r}$ is the natural frequency of a reference mode whose value is the most commensurable to $\omega_{s}$ (i.e., by the Strouhal law $\omega_{n r} \cong 2 \pi \mathrm{St} V_{a v} / D$ ) for a given current flow speed $V_{a v}$ (e.g., the averaged value in the experimental laboratory or actual design). For a given range of $U_{r}$, the maximum and minimum $V$ values are determined based on equation (22) with the chosen $\omega_{n r}$, taking into consideration also the associated $\mathrm{Re}$ number that should remain in the sub-critical range $300<\mathrm{Re}<3 \times 10^{5}$ (see, e.g., Sumer and Fredsoe 1997). By increasing or decreasing $V$, $\omega_{s}$ is then varied through equations (13-16).

\section{Model Validation with Shear7: Single-Mode VIV of TTR}

Prior to investigating the multi-mode VIV of SCRs, a preliminary validation of the presented wake-riser modeling is performed by making a comparison of obtained maximum amplitudes $(A / D)$ in a given $U_{r}$ range $\left[2<U_{r}<10\right]$ with those of Shear7 (v4.5, 2007), in the case of a constant-tension TTR subject to uniform flow. In so doing, the pinned-pinned TTR corresponding to SCR1 $(\Delta=272)$ is considered (Table 2) with three damping ratios $(\xi=.05, .01, .003)$ and thus $S_{\mathrm{G}}(1.133$, $.227, .068)$. For comparison purpose, the geometric nonlinear terms are discarded in the time-domain analysis since Shear7 considers the linear equation of tensioned-beam motion in the 
frequency domain analysis. For a given $V_{a v}=0.4 \mathrm{~m} / \mathrm{s}\left(\omega_{s}=\right.$ $1.308 \mathrm{rad} / \mathrm{s})$, the reference mode is the $4^{\text {th }}$ mode $\left(\omega_{4}=1.461\right.$ $\mathrm{rad} / \mathrm{s}$ ) whose shape is anti-symmetric with respect to middle span, having 4 half-sine waves.

Table 3 Comparison of A/D for TTR due to cross-flow VIV

\begin{tabular}{|c|c|c|c|}
\hline \multirow{2}{*}{$S_{\mathrm{G}}$} & \multicolumn{3}{|c|}{$A / D$} \\
\cline { 2 - 4 } & \multirow{2}{*}{ This study } & \multicolumn{2}{|c|}{ Shear7 } \\
\cline { 3 - 4 } & & $C_{L^{-}}$-Type 1 & $C_{L^{-} \text {-Type } 2}$ \\
\hline 1.133 & 0.394 & 0.438 & 0.427 \\
\hline 0.227 & 1.083 & 1.060 & 0.925 \\
\hline 0.068 & 1.252 & 1.231 & 1.018 \\
\hline
\end{tabular}

A single-mode lock-in analysis based on coupled wakestructural oscillators is standard, exhibiting a jump phenomenon in the amplitude response diagram when varying $U_{r}$ due to the hysteresis effect (Srinil et al. 2008). Herein, the predicted $A / D$ values with wake oscillator and Shear 7 are quantitatively compared in Table 3. In addition, they are compared with experimental data of rigid/flexible cylinders subject to crossflow VIV (Skop and Balasubramanian 1997) in Figure 3 (semi$\log$ scale) which is the so-called Griffin plot. It is worth noting that, in Shear7 analysis (also $\omega_{4}=1.461 \mathrm{rad} / \mathrm{s}$ ), the experimental lift coefficients $C_{L}$-Type 1 and Type 2 of bare cylinders are both considered. They depend on only $A / D$ (Type 1 ) or on both $A / D$ and $U_{r}$ (Type 2). As for the wake oscillator, the $C_{L}$ in equation (1) is thought to be equivalent to $C_{L}$-Type 2 of Shear7 (albeit with different experiment testing) due to the fact that SB empirical wake coefficients depend on both $A / D$ and $U_{r}$.

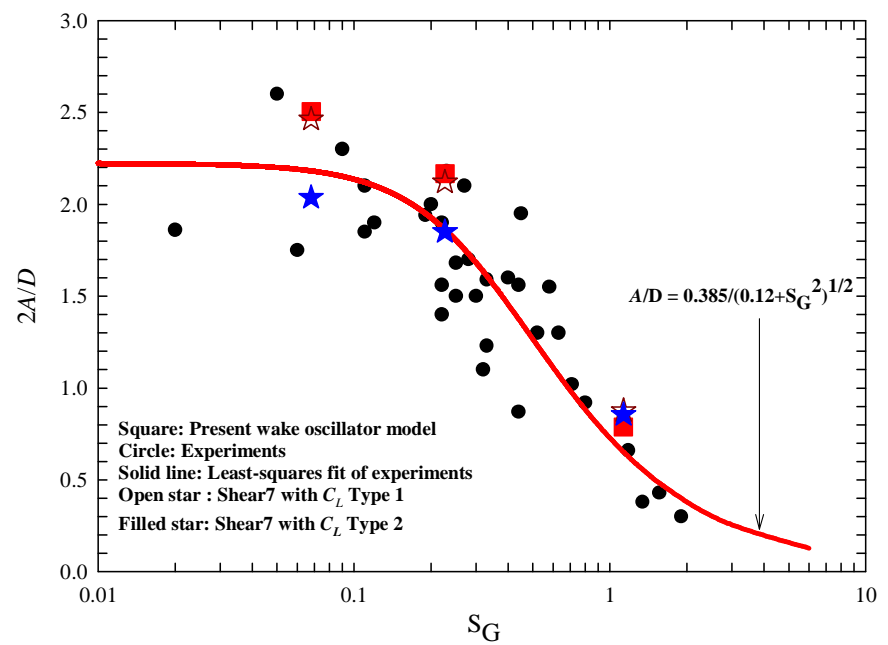

Figure 3 Comparison of $A / D$ with analytical tools and experiments

Overall, good qualitative and quantitative comparison of $A / D$ is seen in both Table 3 and Figure 3 with different massdamping parameters $S_{\mathrm{G}}$. In Table 3 , the Shear7 results with $C_{L^{-}}$ Type 1 provide a more conservative $A / D$ prediction than those with $C_{L}$-Type 2 . When compared with Shear7 results, the wake oscillator accounts for both lower $(\xi=.05)$ and higher $(\xi=$ $.003)$ conservative aspect, depending on the assigned $S_{\mathrm{G}}$.

\section{Nonlinear Multi-Mode VIV Responses of SCR}

To carry out the multi-mode VIV analysis, the considered $N$ potentially vortex-excited modes should include the modes in the neighborhood of the reference mode and the latter. The convergence study of nonlinear responses has to be also made with varying $N$ in the analysis. Herein, by considering SCR1 and SCR2 (Table 2) with $V_{a v}=0.4$ and $1.0 \mathrm{~m} / \mathrm{s}$, the associated $\omega_{s}$ values are 1.308 and $89.76 \mathrm{rad} / \mathrm{s}$, respectively. Thus, with maximum $N=7$, the low- or higher-order modes of interest and relevant natural frequencies in still water $\omega_{n}(\mathrm{rad} / \mathrm{s})$ are given in Table 4, with grey-filled rows denoting reference modes.

Table 4 Natural frequencies of chosen VIV modes for SCRs

\begin{tabular}{|r|r||r|r|}
\hline & SCR1 & & SCR2 \\
\hline$\omega_{2}$ & 1.033 & $\omega_{7}$ & 46.770 \\
\hline$\omega_{3}$ & 1.461 & $\omega_{8}$ & 57.432 \\
\hline$\omega_{4}$ & 1.755 & $\omega_{9}$ & 69.492 \\
\hline$\omega_{5}$ & 2.168 & $\omega_{10}$ & 82.781 \\
\hline$\omega_{6}$ & 2.228 & $\omega_{11}$ & 97.439 \\
\hline$\omega_{7}$ & 2.620 & $\omega_{12}$ & 113.398 \\
\hline$\omega_{8}$ & 2.947 & $\omega_{13}$ & 130.728 \\
\hline
\end{tabular}

Depending on the assigned initial conditions and overall system parameters, numerical simulations of system equations (13-16) are performed to determine the steady-state dynamic responses. Prior to evaluating the relevant $A / D$, it is worth visualizing the time histories associated with each generalized displacement coordinate of riser $\left(f_{n}\right)$, as illustrated in Figure 4 for SCR1 with $N=5(n=2-6)$ and $U_{r}=10$.

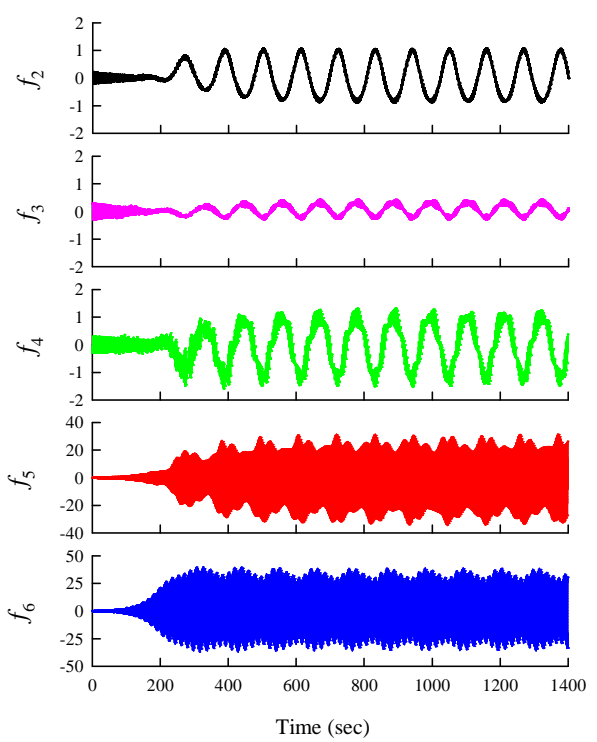

Figure 4 Time series of modal displacement coordinates of SCR1 


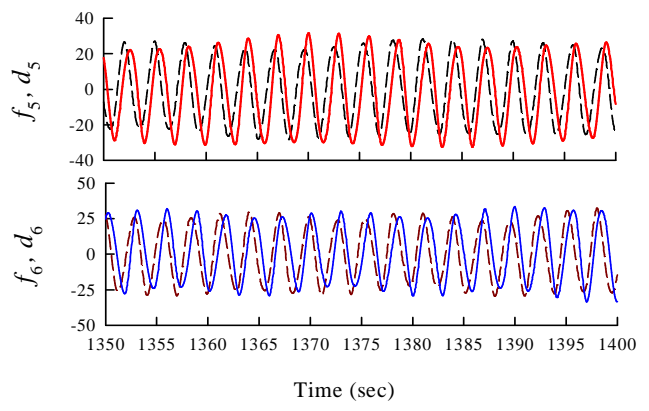

Figure 5 Predominant-mode time histories of riser vs. wake displacement coordinates
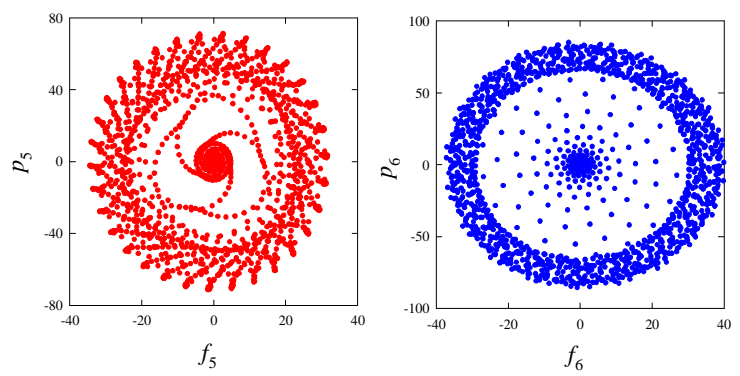

Figure 6 Phase portraits of mode 5 and 6 responses

It can be seen in Figure 4 that the steady-state nonlinear VIV responses (i.e. the limit cycles) occur with more than one predominant mode. Both $f_{5}$ and $f_{6}$ time histories have large magnitude with respect to the others $\left(f_{2}-f_{4}\right)$ and reveal amplitude modulation features as a result of modal interactions. As shown in Figure 5 ( $t=1350-1400 \mathrm{sec}$.), the associated wake coordinates $d_{5}$ and $d_{6}$ (dashed lines) also exhibit the same characteristics as $f_{5}$ and $f_{6}$ (solid lines), with the oscillating wake-riser frequencies being nearly tuned in a 1:1 ratio (i.e. multi-mode lock-in). The phase $\left(f_{n}-p_{n}\right)$ portraits in Figure 6 (starting from transient to steady state) also confirm the periodicity of VIV responses by showing the closed circle orbits.

Numerical integration results in Figures 4-6 highlight how a series of $n$ coupled wake-structure oscillators could be used in the analysis of multi-mode VIV in addition to single-mode VIV (Srinil et al. 2008). As riser responses consist of horizontal and vertical displacements, the resulting maximum amplitude of each modal coordinate $\left(A_{n} / D\right)$ is calculated by

$$
A_{n} / D=\operatorname{Max} \sqrt{\left(f_{n} \phi_{n}\right)^{2}+\left(f_{n} \varphi_{n}\right)^{2}}
$$

where maximum $\phi_{n}$ and $\varphi_{n}$, and $f_{n}$ are determined from the eigenfunction and time series analysis, respectively. To evaluate the root-mean-square (RMS) $A_{n} / D$, spatial maximum $\phi_{n}$ and $\varphi_{n}$ remain fixed, whereas the RMS $f_{n}$ is determined by excluding the initially transient dynamics. In turn, the effective maximum amplitude $A_{R} / D$ of the riser - accounting for overall modal contributions and their actual relative phases in the time series and shape functions - is determined through equation (11).
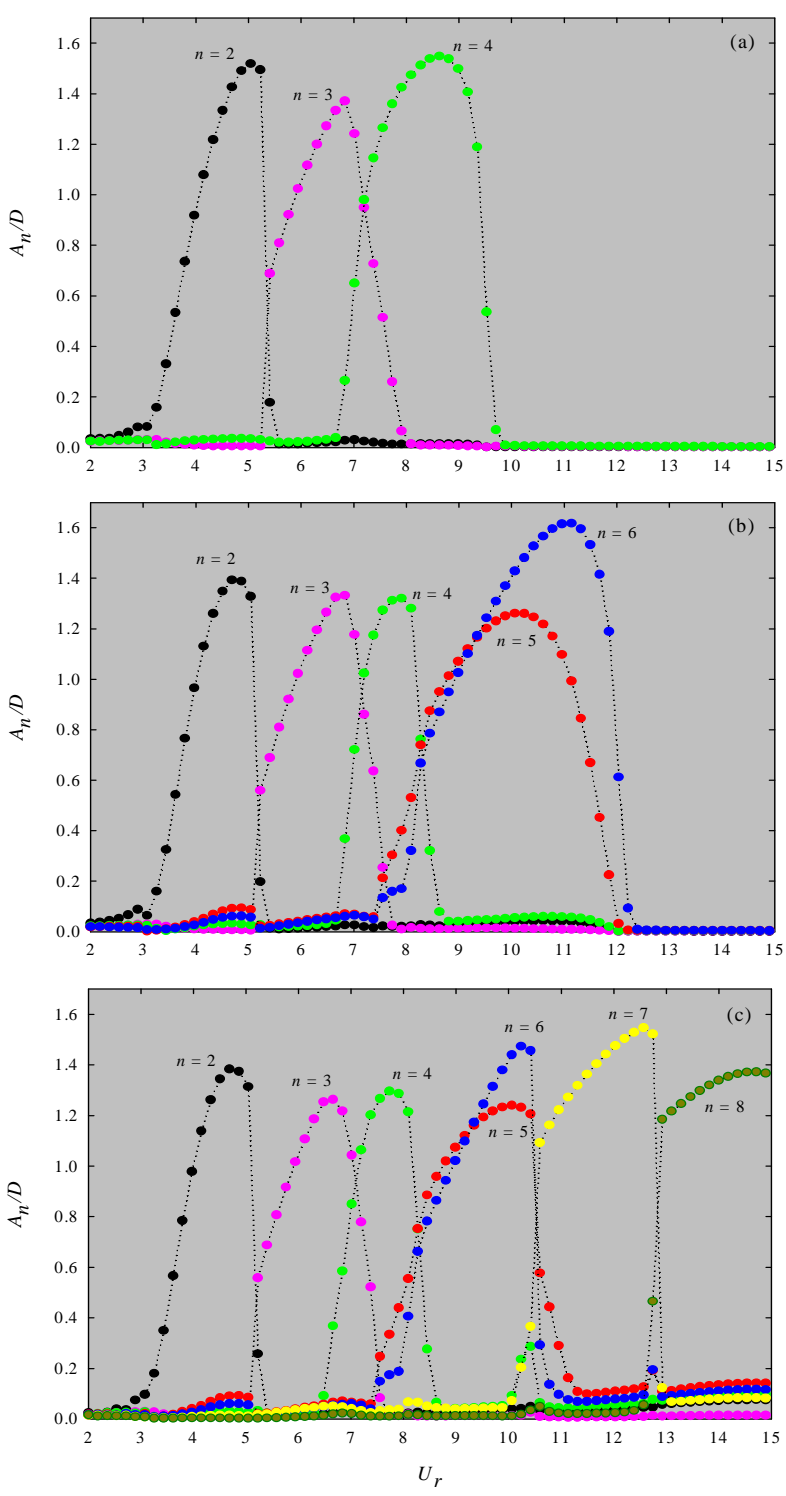

Figure 7 Maximum response amplitudes for SCR1: (a) $N=3$, (b) $N=5$, (c) $N=7$

\section{Multi-Mode Lock-In Behaviors of SCR}

Several experimental and CFD studies have highlighted the multi-mode VIV of straight vertical risers (e.g. Willden and Graham 2004; Trim et al. 2005; Yang et al. 2008). In this study, the multi-mode lock-in behaviors of SCRs subject to transversal uniform current are parametrically investigated. The current velocity $V$ is slowly increased with a small increment $(.04 \mathrm{~m} / \mathrm{s})$ for a given $U_{r}$ range. To comprehend how many DOF are actually needed in obtaining a robust reduced-order solution, it is necessary to perform a convergence study by varying the number of considered $N$ riser modes. By way of examples, the cross-flow VIV of SCR1 (Table 2) is analyzed in Figure 7 for the given range $2<U_{r}<15$. For the sake of comparison in all $N$ 
cases, the $3^{\text {rd }}$ mode is considered as the reference mode (Table 4), the modal damping $\xi$ and wake coefficients $(F, G)$ are fixed. The variation of modal amplitudes $A_{n} / D$ vs. $U_{r}$ is depicted in Figures $7 \mathrm{a}, \mathrm{b}$ and $\mathrm{c}$ for $N=3(n=2-4), 5(n=2-6)$ and $7(n=2$ $8)$, respectively. Moreover, the relevant effective amplitudes $A_{R} / D$ of all combined modes are plotted in Figure 8, together with those of the single-mode $N=1$ solution $(n=3)$.

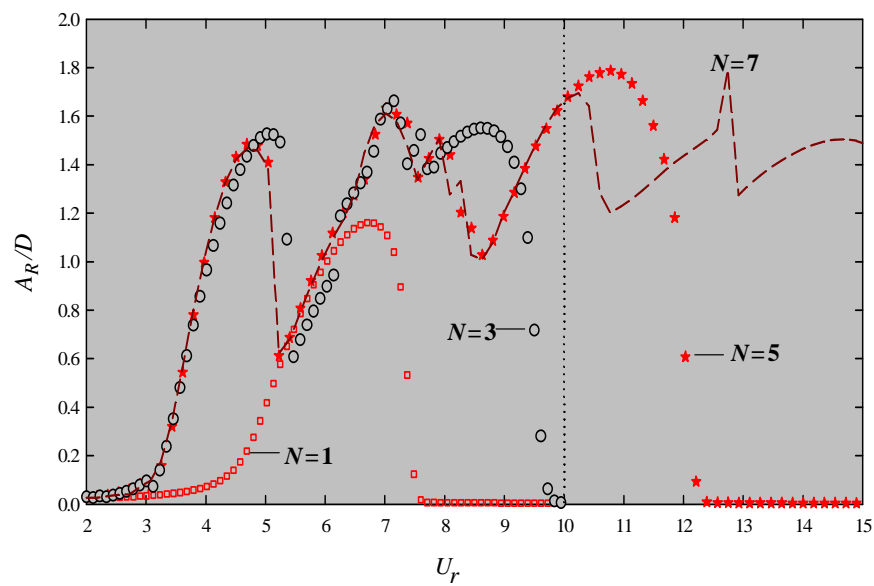

Figure 8 Effective response amplitudes for SCR1

Overall, a jump phenomenon due to hysteresis effect is observed in all $N$ cases (Figure 7). This is typical because of using the nonlinear van der Pol oscillator (Srinil et al. 2008). Depending on modal shape characteristics and system parameters, all riser modal amplitudes reveal self-limiting responses (Figure 7) and entail the maximum $A_{R} / D$ of flexible cylinder in the range of 1 to 2 (Figure 8 ). The $7^{\text {th }}$ mode provides the largest response with $N=7$ (Figure 7c). The observed interesting behaviors are the "switching" and "sharing" of modes in the VIV responses. For instance, with $N=3$ in Figure $7 \mathrm{a}$, the $3^{\text {rd }}$-mode solution prevails after the jump (switching) of the $2^{\text {nd }}$ mode solution occurs at $U_{r} \approx 5$.6. The overlapping (sharing) of modal amplitudes takes place in a particular range of $U_{r}$ as contributions from 2 (Figure $7 \mathrm{a}$ ) or 3 (7b and $7 \mathrm{c}$ ) participating modes are comparably significant. The percent sharing of modes may be low or high depending on the tuning of system natural frequencies (see Table 4). It is seen that the $5^{\text {th }}$ and $6^{\text {th }}$ modes are strongly coupled. Such modes sharing - along with their modal interactions - can also be seen through time histories as illustrated in Figure 4 with $N=5$ and $U_{r} \approx 10$. The $5^{\text {th }}$ and $6^{\text {th }}$ mode responses attain their maximum states at different time instants. These highlight the occurrence of multi-mode lock-in or synchronization whereby the wake/riser oscillating frequencies of each generalized coordinates $\left(f_{n}, d_{n}\right)$ are internally resonant as a nearly-perfect 1:1 ratio (see, e.g., Figure 5).

As regards the convergence analysis, both quantitative and qualitative differences are seen in Figures 7 and 8 with different considered $N$ modes. The single-mode solution seems to be the worst case due to the fact that it cannot capture at all the multimode switching, sharing and interaction features. In addition, the predicted $A_{R} / D$ values are substantially underestimated. With $N=3$, the low reduced-order solution (Figure 7a) is improved, but still providing overestimated $A_{n} / D$ results and broadening the overall lock-in regimes of modes 2-4, with respect to the cases with $N=5$ (Figure $7 \mathrm{~b}$ ) and $N=7(7 \mathrm{c})$. The results converge when further increasing $N$. In both Figures 7 and 8 , considering $N=5$ in the range $2<U_{r}<10$ is seen to be sufficient. To improve the solution when $U_{r}>10$ (see the vertical dotted line in Figure 8) with the minimum $N, 5$ modes with $n=4-8$, instead of $n=2-6$, could be satisfactory.
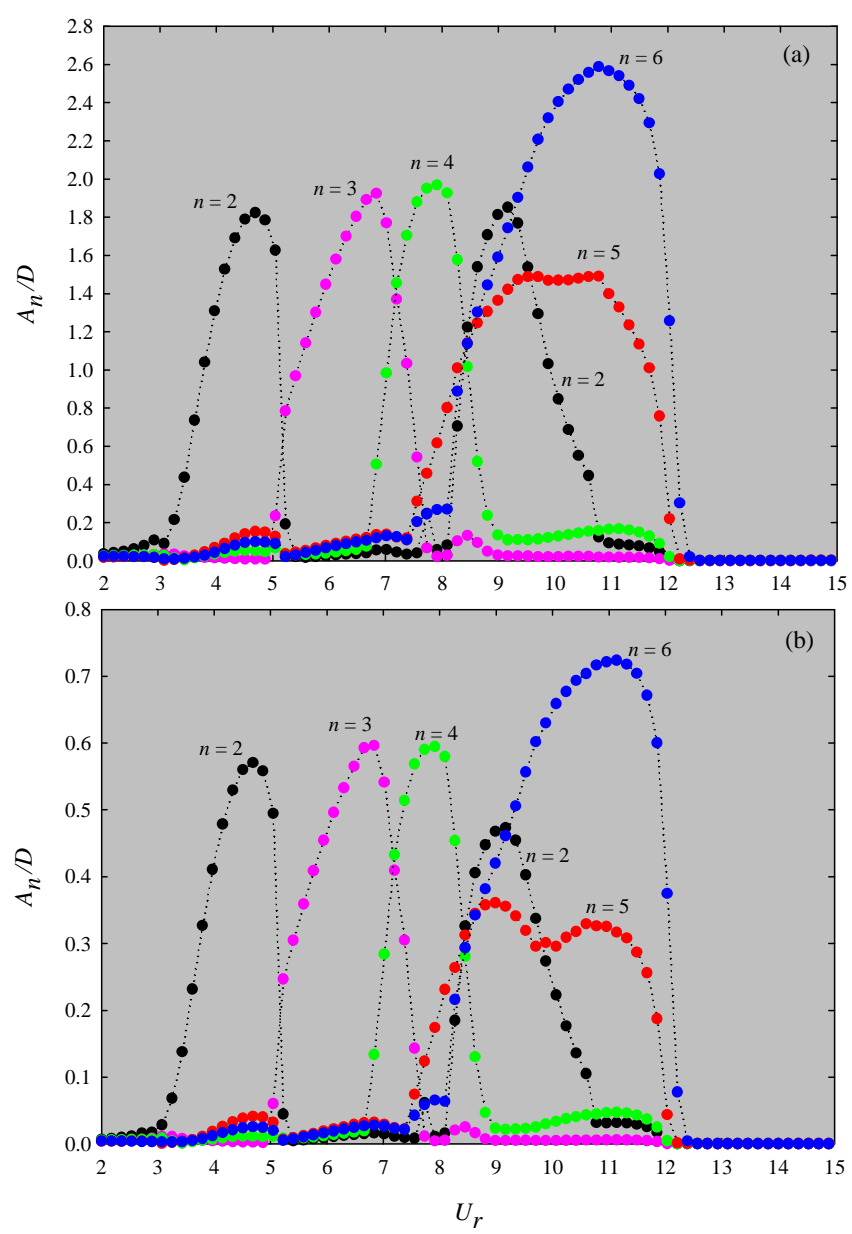

Figure 9 (a) Maximum and (b) RMS response amplitudes for SCR1 with $N=5$ and varying wake coefficients

\section{Influence of Reynolds number}

It is interesting to examine the influence of Re number on the prediction of VIV response amplitudes. In contrast to the results obtained in Figures 7 and 8, the wake coefficients $(F, G)$ are now varied through Equations (6) and (16) with increasing $U_{r}$ or Re (see e.g. Table 1). The SCR1 is again considered with $N=5 \quad(n=2-6)$, and the maximum (9a) and RMS (9b) modal amplitude $A_{n} / D$ results are displayed in Figure 9 in comparison with Figure $7 \mathrm{~b}$. It can be seen that stronger modal interaction takes place in Figure 9. Both maximum and RMS amplitude 
response diagrams exhibit similar features, with the latter also showing two response peaks corresponding to the $5^{\text {th }}$ mode solution. The maximum amplitude $A_{n} / D$ during lock-in reaches the highest value about $2.6 \quad(n=6)$ whereas overall RMS amplitudes are less than 1 . The sharing of 4 modes $(n=2,4,5$, 6 ) in the VIV responses is remarkable in the range $8<U_{r}<9$. In particular, the $2^{\text {nd }}$ modal amplitude participates into the response once again after it previously appears in the lower lock-in bandwidth $\left(3<U_{r}<5\right)$ as in the case of neglected $\mathrm{Re}$ effect (Figure $7 \mathrm{~b}$ ). This $2^{\text {nd }}$ mode is driven possibly due to a socalled multiple internal resonance condition associated with system quadratic/cubic nonlinearities (Srinil et al. 2007). Since the associated natural frequencies of the $2^{\text {nd }}(1.033) 5^{\text {th }}(2.168)$ and $6^{\text {th }}(2.228)$ modes are nearly tuned as 1:2:2 ratio, the nonlinear orthogonality properties of modes are not satisfied (Srinil and Rega 2007a), and the wake nonlinear damping coefficient $(\mathrm{G})$ decreases with Re (Table 1), these modes become strongly coupled in the multi-mode VIV responses.

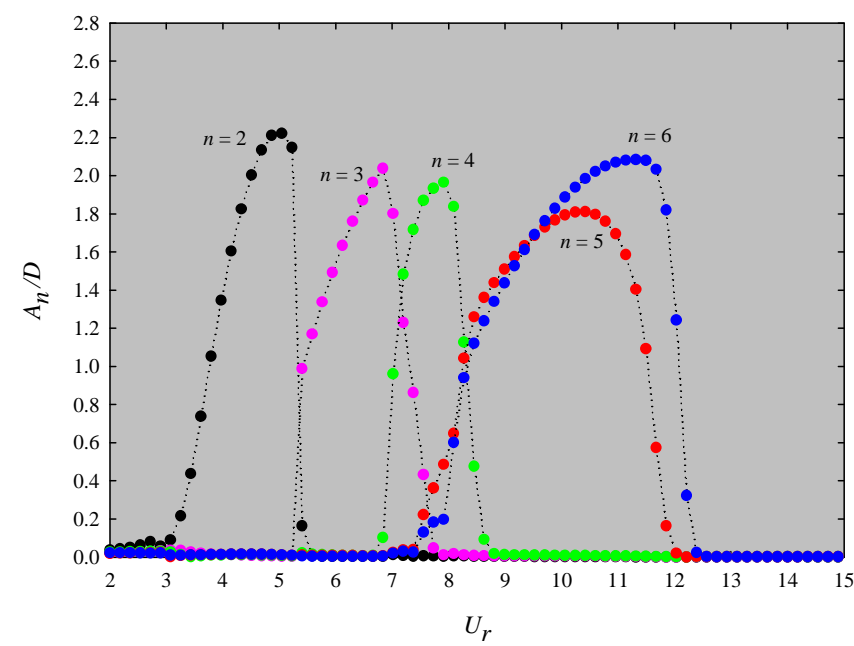

Figure 10 Maximum response amplitudes for SCR1 with $N=5$, varying wake coefficients and linear structural model

\section{Influence of Geometrical Nonlinearities}

The significance of SCR geometrical nonlinearities on the VIV response prediction is now highlighted. By making use of the linear structural modeling with the summation terms in Equation (14) being disregarded, the cross-flow VIV of SCR1 is again analyzed with $N=5$ and the predicted maximum amplitude results are shown in Figure 10 (linear model) in comparison with Figure 9a (nonlinear model). It can be seen that the linear model overestimates (underestimates) the $2^{\text {nd }}$ and $5^{\text {th }}\left(6^{\text {th }}\right)$ mode solutions. In addition, qualitative discrepancy occurs: the second lock-in bandwidth of the $2^{\text {nd }}$ mode response disappears in Figure 10, in contrast to Figure 9a that captures the multiple internal resonances. Therefore, the multi-mode interaction observed in Figure 10 is solely due to the cubic-type nonlinear terms in the wake equations. To accurately evaluate the associated VIV fatigue damage, the predicted vibration amplitudes should rely on the nonlinear structural modeling.

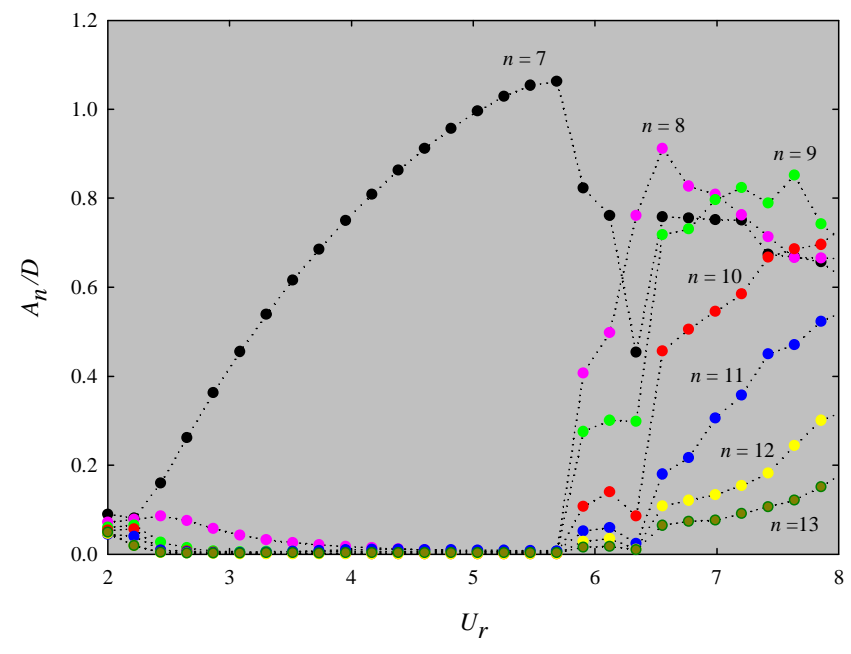

Figure 11 Maximum response amplitudes for SCR2 with $N=7$

\section{High-Order Mode VIV Responses}

The cross-flow VIV responses at higher-order (i.e. $n \geq 10$ ) modes are now discussed by considering the beam-dominant SCR2 (Tables 2 and 4). It is worth noting that the corresponding natural frequencies of SCR2 $(\Delta=21)$ are quite widely spaced, whose values are much higher than those of SCR1 $(\Delta=272)$ due to greater bending contributions. By considering $2<U_{r}<8$ with fixed wake coefficients and the $10^{\text {th }}$ mode being the reference mode, the predicted maximum amplitudes of considered 7 modes $(n=7-13)$ are depicted in Figure 11. In contrast to Figure 7, there is no clear jump phenomenon in Figure 11. The $7^{\text {th }}$ mode response prevails over a wide $U_{r}$ range with maximum amplitude reaching $A_{7} / D \approx 1.1$. The relatively strong modal interaction occurs when $U_{r}>6$ with higher-order multi modes playing a meaningful role. To gain further insight into the corresponding nonlinear dynamics, the time series of all modal coordinates are plotted in Figure 12 with $U_{r} \approx 5.9$ (left column) and $U_{r} \approx 7.6$ (right column). It is seen that the responses of $U_{r} \approx$ 5.9 are quite steady and periodic with 3 sharing modes $(n=7-9)$ whereas those of $U_{r} \approx 7.6$ are unsteady, highly- modulated and chaotic with 5 sharing modes $(n=7-11)$. The VIV responses seem to be more fluctuating at higher $U_{r}$ which involves higherorder modes. Consequently, the multi-harmonic time histories of riser/wake contain many frequencies and the perfect lock-in or 1:1 resonant condition does not seem to occur.

The occurrence of unsteady, highly amplitude-modulated and multi-harmonic cross-flow responses of the same riser (SCR2) subject to uniform flow perpendicular to its curvature plane has been experimentally observed in the STRIDE project (Moe et al. 2004). Also, the measured maximum amplitude $A / D$ was also in the range of 0.5-1 depending on the excited mode. These outcomes are in good qualitative agreement with the analytical prediction in Figures 11 and 12. Because SCR contains high modal density owing to the effect of initial curvatures, the multi-mode, multi-harmonic or chaotic VIV responses with high temporal variation are feasible. 

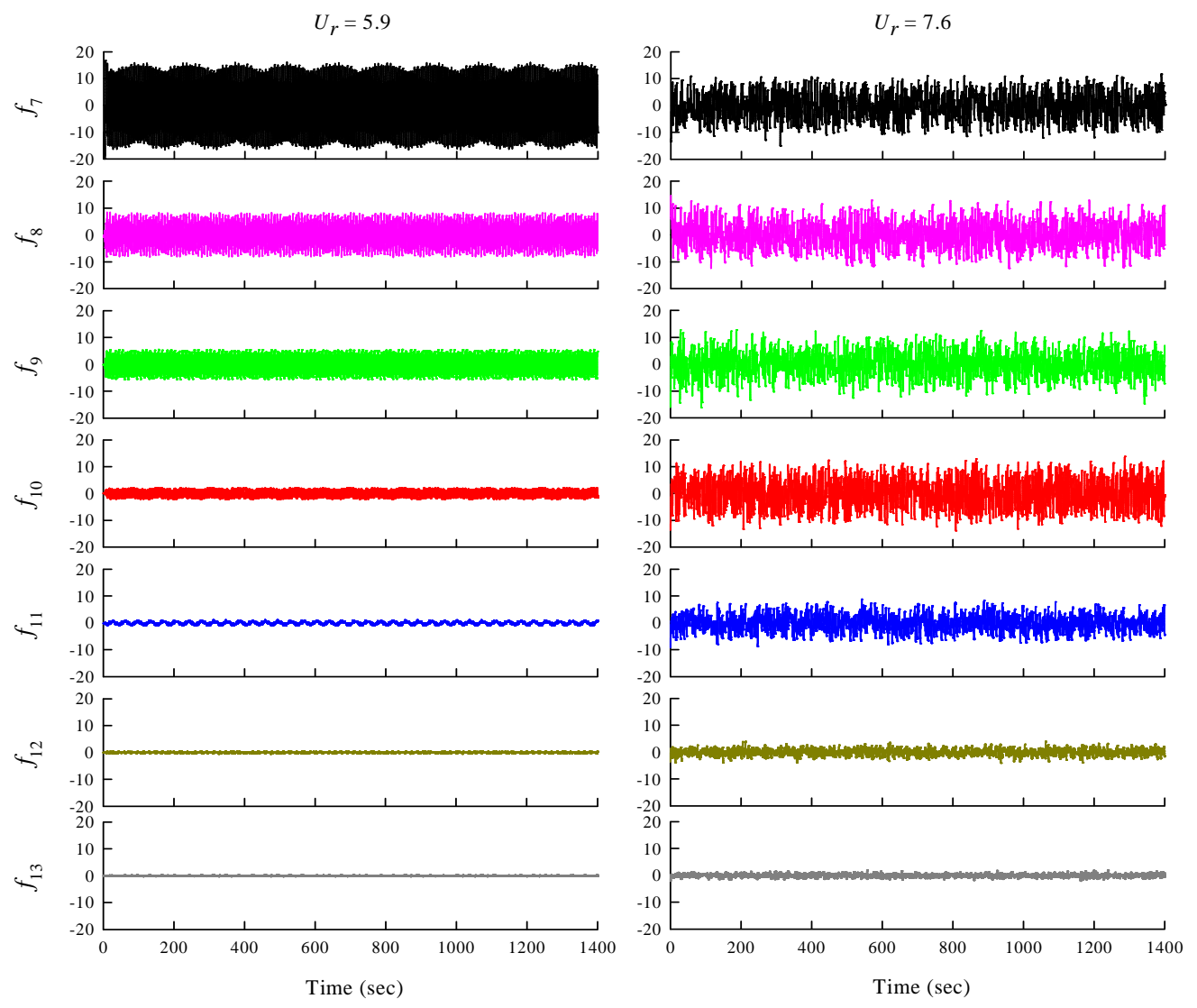

Figure 12 Time series of modal displacement coordinates of SCR2

\section{CONCLUSIONS}

A novel reduced-order fluid-structure interaction model capable of analyzing the multi-mode cross-flow VIV of catenary riser subject to the ocean current has been developed and systematically investigated. The incoming flow has been assumed to be steady, uniform, unidirectional and perpendicular to the riser plane of initial curvatures. The equations of riser motion are based on a pinned-pinned beam-cable modeling with bending/axial extensibility effect and geometrical nonlinearities. A distributed van der Pol wake oscillator has been utilized as the empirical VIV forcing function and modified to interact spatially and temporally with the horizontal/vertical dynamic displacements of riser. Essentially, both structural and fluid models account for the effect of initial curvatures of the elastic inclined flexible cylinder. Depending on the number of vortexexcited modes, a series of nonlinear coupled riser-wake differential equations have been derived and solved by numerical time integrations, with the aim of evaluating the associated maximum/RMS response amplitudes of riser. The single- and multi-mode lock-in analysis has been performed by varying the reduced flow velocity parameter. The fundamental analysis results, in the case of single-mode cross-flow VIV of a straight uniformly-tensioned riser, are in good agreement with those predicted by Shear7 and experimental data.
For catenary risers, the modal convergence study has been conducted by varying the number of considered planar modes. Results highlight both quantitative and qualitative discrepancies with a single-mode (or a very low DOF) solution. Depending on the system fluid-riser parameters, the prevalence of tension or bending rigidity, the relationship between vortex and natural frequencies and the assigned initial conditions, the multi-mode solution provides several insights into the VIV behaviors of catenary risers. These include the occurrence of multi-mode lock-in, switching, sharing and interaction features in the amplitude response diagrams. The sharing of modes in VIV is also observed through the associated time histories. When also taking into consideration the effect of Re number through the underlying wake oscillators, some quantitative and qualitative behaviors remarkably change with regard to the multi-mode interaction. In particular, when increasing the flow speed, the occurrence of multiple internal resonances between higher and lower modes is meaningful. This feature is associated with geometrical nonlinearities and thus is not captured by the linear structural model. Finally, the analysis of high-order mode VIV highlights the highly-modulated multi-harmonic responses with strong modal interactions. The associated time signals are chaotic and contain non-resonant (non-lock-in) oscillating frequencies with a high temporal variation. 
In spite of making available the general reduced-order fluid-structure interaction model for multi-mode cross-flow VIV analysis of catenary risers, as well as top-tensioned risers (Srinil et al. 2009), subject to uniform current flow and addressing some interesting observations, the present modeling and analysis outcomes need further verification with respect to recent experimental testing results and industrial analytical tools. As regards VIV forcing function, the nonlinear wake oscillator and pertinent empirical coefficients need further improvement towards practical applications. These include the cases of multi-mode interaction between cross-flow and in-line VIV, sheared flow or flow being non-perpendicular to the riser plane of initial curvatures, and travelling wave characteristics along riser. Moreover, it is worth carrying out a new series of curved riser VIV experiments by also recognizing the inherent effect of cylinder initial curvatures, the Re number and multimode dependence in VIV responses.

\section{ACKNOWLEDGEMENT}

The authors gratefully acknowledge the funding support from the Knowledge Transfer Partnerships (KTP) in the UK.

\section{REFERENCES}

Cunha, L. D., Pesce, C. P., Wanderley, J., Fujarra, A. L. C., 2006, "The robustness of the added mass in VIV models," in Proceedings of the $25^{\text {th }}$ OMAE, Hamburg, Paper No.92323.

de Lima, A. A., Meneghini, J. R., Mourelle, M., Casaprima, E., Flatschart, R. B., 2007, "Numerical investigation of vortex-induced vibration of a marine SCR," in Proceedings of the $26^{\text {th }}$ OMAE, San Diego, Paper No.29269.

Facchinetti, M., de Langre, E., Biolley, F., 2004, "Coupling of structure and wake oscillators in vortex-induced vibrations," Journal of Fluids and Structures 19, pp. 123-140.

Gabbai, R. D., Benaroya, H., 2005, "An overview of modeling and experiments of vortex-induced vibration of circular cylinders," Journal of Sound and Vibration 282, pp. 575-616.

Govardhan, R. N., Williamson, C. H. K., 2006, "Defining the modified Griffin plot in vortex-induced vibration: revealing the effect of Reynolds number using controlled damping," Journal of Fluid Mechanics 561, pp. 147-180.

Hatton, S., Willis, N., 1998, "Steel catenary risers for deepwater environments," in Proceedings of the OTC, Paper No.8607.

Kim, W. J., Perkins, N. C., 2002, "Two-dimensional vortexinduced vibration of cable suspensions," Journal of Fluids and Structures 16, pp. 229-245.

Lie, H., Larsen, C. M., Tveit, O., 2001, "Vortex induced vibration analysis of catenary riser," in Proceedings of the OTC, Paper No.13115.

Miliou, A., Sherwin, S. J., Graham, J. M., 2003, "Fluid dynamic loading on curved riser pipes," Journal of Offshore Mechanics and Arctic Engineering 125, pp. 176-182.
Moe, G., Teigen, T., Simantiras, P., Willis, N., Lie, H., 2004, "Predictions and model tests of a SCR undergoing VIV in flow at oblique angles," in Proceedings of the $23^{\text {rd }}$ OMAE, Vancouver, Paper No.51563.

Parkinson, G., 1989, "Phenomena and modeling of flowinduced vibrations of bluff bodies," Progress in Aerospace Sciences 26, pp. 169-224.

Skop, R. A., Balasubramanian, S., 1997, "A new twist on an old model for vortex-excited vibrations," Journal of Fluids and Structure 11, pp. 395-412.

Srinil, N., Rega, G., Chucheepsakul, S., 2007, "Two-to-one resonant multi-modal dynamics of horizontal/inclined cables. Part I: Theoretical formulation and model validation," Nonlinear Dynamics 48, pp. 231-252.

Srinil, N., Rega, G., 2007a, "Two-to-one resonant multi-modal dynamics of horizontal/inclined cables. Part II: Internal resonance activation, reduced-order models and nonlinear normal modes," Nonlinear Dynamics 48, pp. 253-274.

Srinil, N., Rega, G., 2007b, "The effects of kinematic condensation on internally resonant forced vibrations of shallow horizontal cables," International Journal of Nonlinear Mechanics 42, pp. 180-195.

Srinil, N., Wiercigroch, M., O’Brien, P., Lane, M., 2008, “New model for vortex-induced vibration of catenary riser," in Proceedings of the $8^{\text {th }}$ ISOPE-PACOMS, Bangkok, pp. 129-136.

Srinil, N., Wiercigroch, M., O'Brien, P., 2009, "Nonlinear multi-mode interactions in subsea risers undergoing vortex-induced vibrations," in Proceedings of the $19^{\text {th }}$ ISOPE, Osaka, Paper No. TPC-149.

Sumer, B. M, Fredsoe, J., 1997, Hydrodynamics around Cylindrical Structures, World Scientific, Singapore.

Swithenbank, S. B., Vandiver, J. K., Larsen, C. M., Lie, H., 2008, "Reynolds number dependence of flexible cylinder VIV response data," in Proceedings of the $27^{\text {th }}$ OMAE, Estoril, Paper No.57045.

Trim, A. D., Braaten, H., Lie, H., Tognarelli, M. A., 2005, "Experimental investigation of vortex-induced vibration of long marine risers," Journal of Fluids and Structures 21, pp. 335-361.

Vandiver, J. K., et al., 2007, "User Guide for Shear7 v.4.5," Atlantia Offshore Limited.

Vandiver, J. K., Gonzales, E. C., 1997, "Fatigue life of catenary risers excited by vortex shedding," in Proceedings of the $8^{\text {th }}$ BOSS, Delft.

Violette, R., de Langre, E., Szydlowski, J., 2007, "Computation of vortex-induced vibration of long structures using a wake oscillator model: comparison with DNS and experiments," Computers and Structures 85, pp. 1134-1141.

Willden, R., Graham, J. M. R., 2004, "Multi-modal vortexinduced vibrations of a vertical riser pipe subject to a uniform current profile," European Journal of Mechanics B/Fluids 23, pp. 209-218.

Yang, G., Frank, W. R., Campbell, R. B., Slocum, S. T., 2008, "VIV model test data comparison with Shear7 V4.5," in Proceedings of the $27^{\text {th }}$ OMAE, Estoril, Paper No.57108. 